\title{
Advancing Computational Approaches for Study and Design in Catalysis
}

\author{
Sandra Luber* \\ Werner Prize 2018
}

\begin{abstract}
Our group deals with the development of computational methods and their application to complex systems and processes. Emphasis is laid on accurate approaches derived from quantum mechanics, which we have used to investigate challenging questions in an interdisciplinary field encompassing chemistry, biology, physics, and materials science. In this review, we describe our recent activities for advancing computational approaches in catalysis. Moreover, our work for the study and design of catalysts for solar light-driven water splitting is summarized. The latter is a promising approach to face the world's increasing demand for sustainable energy storage and conversion. Emphasis is put on forefront methods for highly accurate electronic structure, sophisticated inclusion of environmental and dynamic effects and the derivation of structure-activity relationships for informed in silico design. This paves the way for the development of more efficient catalysts in close collaboration with experimental groups.
\end{abstract}

Keywords: Catalysis · in silico design · Molecular dynamics · Solvent effects · Transition metals · Water splitting

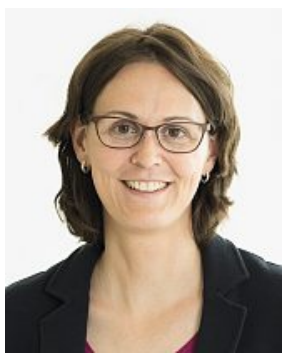

Sandra Luber received a MSc degree in chemistry at ETH Zurich in 2007 and a $\mathrm{PhD}$ degree in (relativistic) quantum chemistry at ETH Zurich in 2009 under the supervision of M. Reiher. For postdoctoral stays, she joined M. Zavolan's group at Biozentrum at the University of Basel (2010) and V. Batista's group at Yale University (2010-2011). Afterwards she worked for BASF SE (2012) and became project group leader with J. Hutter at the University of Zurich where she finished her habilitation thesis in 2016. Since 2017, she has been SNSF profes-

${ }^{\star}$ Correspondence: Prof. S. Luber

Department of Chemistry C

University of Zurich

Winterthurerstrasse 190,

$\mathrm{CH}-8057$ Zurich

E-mail: sandra.luber@chem.uzh.ch sor at the University of Zurich. Research awards include the IBM Research Prize for Computer Modelling and Simulations in Chemistry, Biology, and Materials Science and the ETH medal for an outstanding $\mathrm{PhD}$ thesis. Moreover, she has been the first theoretician to receive the Clara Immerwahr Award and the first female scientist to obtain the Hans G. A. Hellmann Award (awarded annually since 1999) and the Robin Hochstrasser Young Investigator Award for chemical physics. Her research deals with the development and application of static and dynamic (mainly $a b$ initio) computational methods at the interface of chemistry, biology, materials science, and physics. (Image: Jos Schmid)

\section{Introduction}

Sunlight is the largest, freely available energy source on earth, and extensive research has been carried out in order to efficiently use it in a wide variety of processes and applications. In view of the increasing worldwide energy demand, which is becoming more and more serious due to the expected shortage of fossil fuels and related environmental problems, efforts have been put into the conversion and storage of light energy in easily accessible fuels such as molecular hydrogen. The latter can be achieved by water splitting catalysis where sunlight is employed to split water into molecular hydrogen and molecular oxygen. Capturing solar light by means of photosensitizers and using suitable catalysts, water is oxidized, thus generating molecular oxygen as well as protons and electrons (water oxidation). The latter are converted into molecular hydrogen (water reduction) with the help of water reduction catalysts.

Despite huge research efforts, an efficient stable artificial water splitting device is still sought after. In particular the water oxidation reaction is one of the main limiting parts in the overall process. In order to develop new improved water oxidation catalysis in an informed way, in-depth knowledge about the underlying processes and factors influencing the catalytic activity is essential. Experiments allow important information to be gained and various spectroscopic techniques provide a way to elucidate the behaviour of catalysts. Nevertheless, it is often difficult or impossible to get accurate information about every step of the catalytic cycle since intermediates are short-lived and therefore rarely accumulate. Simulations are an additional tool not only to interpret experiments but also to provide further information not accessible from measurements. For understanding at an atomistic level, methods based on quantum mechanics have been proven to be highly useful.

In the following, we describe our efforts for advancing computational approaches in this field (see section 2 and Fig. 1 for an overview about our research directions for methods in catalysis). In section 3 , we summarize our research for the study of bio-inspired cubane catalysts for water oxidation, followed by a short description of our work on in silico design in section 4. A conclusion can be found in section 5 . 
Fig. 1. Overview about research directions in our group for computational methods in catalysis and their comparison to experimental data.

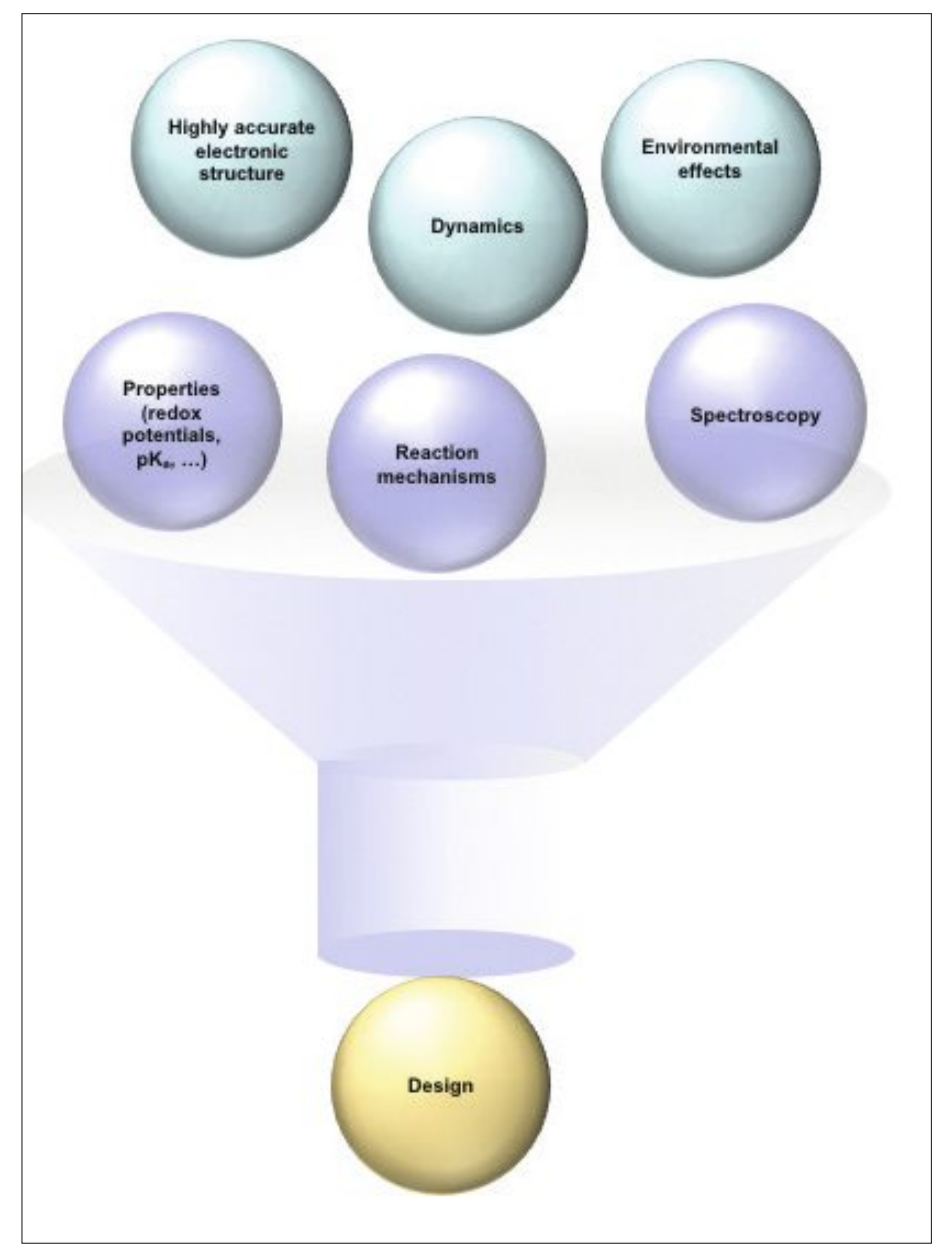

\section{Advancing Computational Approaches in Catalysis}

For a thorough understanding of the functioning of catalysts, it is necessary to have detailed knowledge about their structure, dynamics, and pathways associated with the catalytic activity, i.e. catalytic cycles as well as possible side and deactivation pathways.

The standard approach to elucidate these aspects relies on static computational methods employing Kohn-Sham density functional theory (DFT). The latter usually provides the best compromise between accuracy and efficiency and is the by far most commonly used electronic structure method. Nevertheless, there are shortcomings. For catalytic systems, in particular the ones involving (strongly) correlated transition metals, DFT often leads to, e.g. wrong spin splitting energetics. In order to ameliorate that, multiconfigurational wavefunction-based methods can be applied. Among various approaches available, we have adopted e.g. the complete active space self-consistent field (CAS-SCF) method [and its extension to perturbation theory (CAS-PT2)] to a water oxidation catalyst (WOC) with a ruthenium metal centre (see Fig. 2). Since the number of electrons and orbitals which can be treated with those conventional approaches is limited to active spaces, which may already be too small for systems with one transition metal centre (for instance, if the electronic structure of the ligands plays an important role), we currently apply emerging forefront methods such as CAS quantum Monte Carlo ${ }^{[1]}$ (collaboration with G. LiManni and A. Alavi, MPI Stuttgart). This allows to obtain a highly accurate description of the electronic structure and spin state energetics of involved catalysts such as cubanes featuring four transition metal centres.

For insight into the electronic and nuclear structure of catalysts and their reaction mechanisms, geometry optimizations (i.e. assuming a temperature of $0 \mathrm{~K}$ ) are usually carried out providing structures for the starting, transition, and product states of the reaction of interest. Free energies are standardly calculated using normal mode analysis and the ideal gas, rigid rotor, harmonic oscillator approximation. This approach is computationally rather inexpensive but provides an overall static picture. For instance, compounds may have several conformational isomers which may not be found if only one geometry optimization is carried out. Moreover, the result of the geometry optimization depends on the starting guess structure for that calculation, thus leading to a structure not necessarily being the most representative one for the system and process studied. Generally, molecules behave in a dynamic manner at ambient conditions, which is not accounted for in a static approach. An alternative to that is DFT-based molecular dynamics (MD) [usually referred to as ab initio MD (AIMD)], which treats the electronic structure with DFT and the nuclei classically. ${ }^{[6]}$ This approach has the advantage that the conformational phase space can be explored by treating systems at (more realistic) conditions such as the ones used in experiments. Although leading generally to a higher computational effort than the static approach, and up to now rather rarely used in the field of catalysis, this approach has been shown to be highly valuable. Examples from our work will be given in section 3 (e.g. the discovery of the flexibility of bio-inspired cubane catalysts and associated properties facilitating water oxidation).

The virtue of a dynamic treatment is especially obvious when it comes to the consideration of environmental effects. For water splitting, the surrounding water naturally plays an important role as a solvent and substrate for water oxidation. In calculations, the solvent environment is commonly considered via solvent continuum models, which mimic average solvent effects and are fitted for certain test sets and computational settings (basis set, exchange-correlation density functional), mostly for organic neutral molecules. Directed bonds, e.g. hydrogen bonds, are not well described in currently prevailing solvent continuum models. In order to improve the latter, explicit water molecules may be included into the computational model. Then the question arises how many solvent molecules should be considered and where they should be located. This is especially relevant in the static approach relying on geometry optimizations, which introduces a bias resulting from the starting structure for the optimization. ${ }^{[7]}$ With increasing numbers of solvent molecules, this approach reaches its limitation. ${ }^{[5,7-9]}$ AIMD allows sampling over different configurations and a sophisticated description of condensed phase systems (such as liquids), usually by means of periodic boundary conditions (see Fig. 2). For water, for instance, the dynamics of the liquid, hydrogen bonding networks and associated proton transfers are complex and significantly influence the behaviour of the catalyst. This has already been found for rather simple reactions such as ligand-exchange reactions. ${ }^{[5]}$ Investigating such reactions at a cubane WOC (vide infra), we recently compared reaction energies (mainly electronic energy differences) obtained from static approaches relying on solvent continuum models (COSMO, COSMO$\mathrm{RS}^{[10-12]}$ ) or explicit solvation derived from 


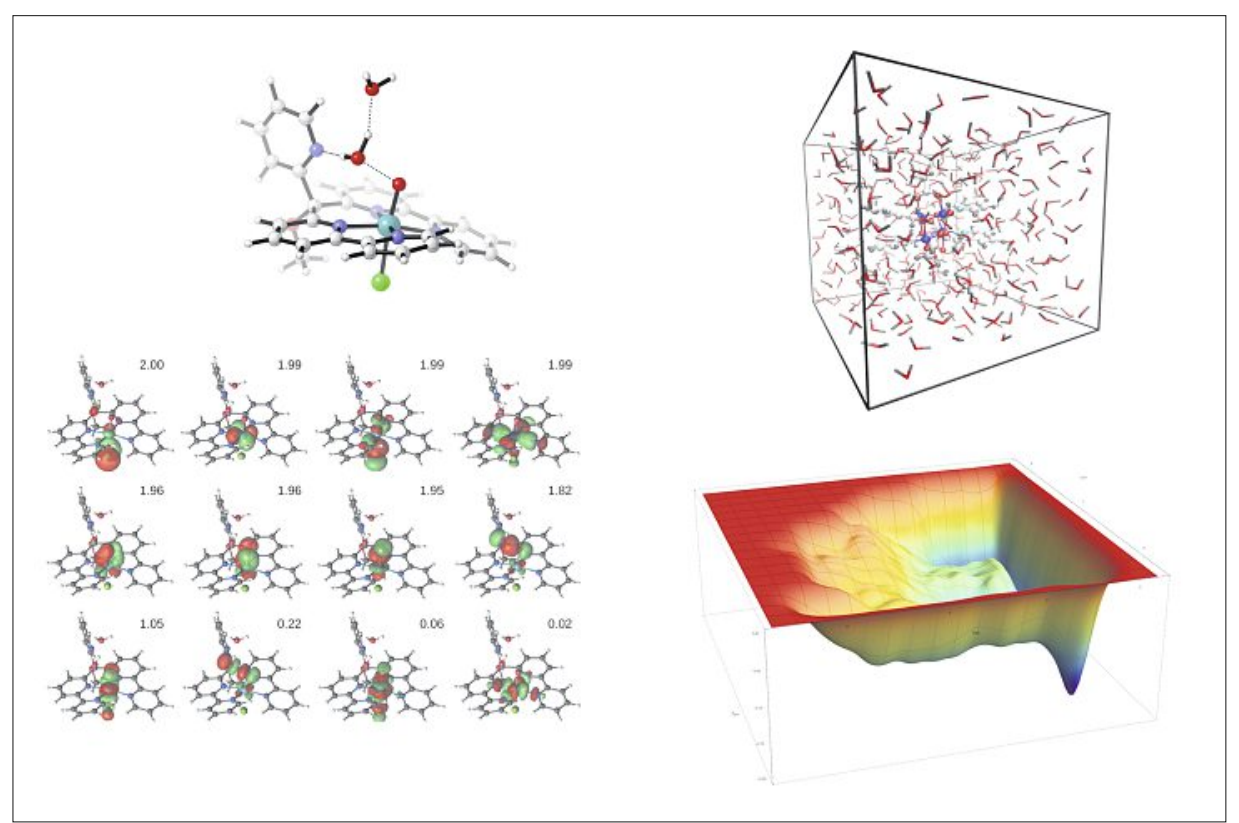

Fig. 2. Left-hand side: Transition state structure of the water nucleophilic attack at a Ru-based WOC (top) $)^{[2,3]}$ and corresponding CAS $(17,12)$ active space with natural occupation numbers ${ }^{[4]}$ (bottom); Right-hand side: Co(॥)-cubane WOC in water solution (top; adapted from ref. [5]) and free energy surface from a metadynamics run for ligand-exchange reactions of said Co(॥)-based WOC (bottom).

snapshots from a DFT-MD or quantum mechanics/molecular mechanics simulation to the ones obtained entirely from DFTMD. ${ }^{[5]}$ In contrast to the results obtained by means of COSMO-RS, the ones computed with the help of the COSMO model were in significantly worse agreement with reaction energies from other approaches, indicating that the popular COSMO solvent continuum model should be applied with care. Non-negligible differences between the two solvent continuum models were observed in the surface charge density maps of lanthanide-containing cubanes as well. ${ }^{[7]}$ It is worth mentioning that not only the water solvent influences the catalytic processes. Often, there are, among others, counter ions in the reaction mixture and, in case of chemical oxidation, oxidizing agents and their reduced products. Moreover, buffer molecules are usually required, which may directly interact with the catalyst (see section 3). ${ }^{[13]}$

In order to improve catalytic efficiency in an informed way, it is necessary to have knowledge about the detailed structure of the catalyst and its behaviour. Reaction mechanisms can be analysed by computing (free) energies of each state occurring during the catalytic cycle leading to information about the associated thermodynamics. Calculation of barriers connecting catalytic states further provides valuable knowledge about the kinetics of the process. In order to elucidate free energies and barriers with AIMD, enhanced sampling techniques such as metadynamics ${ }^{[14]}$ can be applied where the solvent is automatically treated in a dynamic way. This allows access to AIMD-based protocols for the thermodynamics and kinetics of catalytic processes beyond the conventional static picture as well as relevant side reactions such as ligand-exchange reactions (see Fig. 2). ${ }^{[5]}$ For proton coupled electron transfers, which occur frequently in catalytic processes, we have recently presented a thermodynamic integration-based scheme in order to obtain dehydrogenation free energies $^{[15]}$ based on previous work by Sprik and co-workers. ${ }^{[16]}$ This paves the way for calculation of redox potentials and $\mathrm{pKa}$ values, which are important quantities for many research areas in order to elucidate chemical structures and reaction mechanisms in comparison with experimental data. Due to the strong dependence of such calculations on the description of the solvent environment, AIMD-based methods are a promising way to systematically improve such calculations contrary to most of the traditional approaches employing solvent continuum models.

Another vital direction in our research is computational spectroscopy (see CHIMIA 2018, 72, 328 for a review about our work on vibrational spectroscopy[17]). Spectroscopy is indispensable for elucidating catalytic processes. Simulations facilitate not only the interpretation of experimental results, they also provide a way to compare computational results to experimental data, thus allowing to exclude certain scenarios and guide the direction of further (computational) research. During the last five years, we have focused on sophisticated approaches for inclusion of solvent and dynamic effects in spec- troscopic calculations, mainly based on AIMD, and the calculation of condensed phase systems, e.g. molecules in solution or adsorbed on surfaces. ${ }^{[18]}$ Examples include novel analysis approaches and local properties of complex (periodic) systems using DFT embedding techniques ${ }^{[19]}$ and forefront methods for Raman (optical activity), ${ }^{[18,20-23]}$ vibrational circular dichroism, ${ }^{[18]}$ and interface-sensitive sum frequency generation (SFG) ${ }^{[24]}$ spectroscopy. This has led to the first Raman optical activity spectra from AIMD and the first SFG spectrum involving a semiconductor surface entirely calculated with AIMD, which may be of special interest in the future for obtaining information about molecular catalysts absorbed on surfaces, e.g. like the one studied in ref. [25]. Moreover, chiral ionic liquids ${ }^{[26]}$ and photoinduced proton coupled electron transfer ${ }^{[27]}$ have been studied. For transition metal-containing complexes such as the WOCs discussed in section 3, X-ray absorption spectroscopy is a pivotal tool to get information, e.g. about oxidation states of transition metal centres and their ligand environment. In this context, we have developed efficient refinement procedures to improve the agreement of simulated and experimental data by means of calculated extended X-ray absorption fine structure spectra and DFT[28] (and Mössbauer parameters ${ }^{[29]}$ ). In addition, X-ray absorption near edge structure results have been used to study the WOCs discussed in the next section. ${ }^{[13,30,31]}$

\section{Exploring the Secrets of Bio- inspired Cubane Water Oxidation Catalysts}

A paradigm for efficient water oxidation is nature's photosystem II. Water oxidation is carried out by means of the oxygen-evolving complex (OEC), which has a heterocubane structure featuring three redox-active manganese centres and one redox-inert calcium metal centre connected via oxo bridges, complemented by a fourth 'dangling' manganese metal centre (see Fig. 3). This special structure has inspired many researchers world-wide in their search for new efficient artificial WOCs. Up to now, no stable active artificial Mnbased cubane WOC has been reported. In collaboration with the experimental group of G. R. Patzke (University of Zurich), the first Co(II)-based cubane WOCs have been presented. These are some of the rare stable and active artificial cubane WOCs and open up an exciting opportunity to thoroughly elucidate factors determining water oxidation activity. The first $\mathrm{Co}$ (II)based cubane WOC $\left\{\mathrm{Co}_{4}(\mathrm{OR})_{4}\right\} \quad(\mathrm{R}=$ hmp) was presented in 2013[30] (see Fig. 3), followed by lanthanide (Ln)-containing 
cubane WOCs $\left\{\mathrm{Co}_{3} \mathrm{Ln}(\mathrm{OR})_{4}\right\}^{[13]}(\mathrm{Ln}=\mathrm{Er}$, $\mathrm{Tm}, \mathrm{Yb}, \mathrm{Ho}$; see Fig. 3), which are more efficient WOCs than the $\left\{\mathrm{Co}_{4}(\mathrm{OR})_{4}\right\}$ cubane. Within the series of $\left\{\mathrm{Co}_{3} \mathrm{Ln}(\mathrm{OR})_{4}\right\}$ cubanes, the WOC with erbium as redox-inert metal centre gives rise to the highest catalytic activity whereas thulium leads to the lowest catalytic activity.

In order to develop improved cubane WOCs, knowledge about the water oxidation mechanism is indispensable. This makes high-level calculations highly valuable, in particular since it has been very challenging to get experimental data for intermediates occurring during the catalytic cycle. In order to achieve a sound description of the catalytic cycle, the first step is to determine the catalytic resting state, which has not necessarily the same structure as the one obtained from X-ray crystallography (if available). We thus investigated ligand-exchange reactions of the Co-cubane WOCs in solution with a wide range of methods (see section 2) starting from computationally inexpensive geometry optimizations utilizing solvent continuum models up to computationally demanding AIMD-based approaches including metadynamics. ${ }^{[5,13]}$ These calculations made clear that the $\left\{\mathrm{Co}_{3} \operatorname{Er}(\mathrm{OR})_{4}\right\}$ cubane has a more flexible ligand shell than the $\left\{\mathrm{Co}_{4}(\mathrm{OR})_{4}\right\}$ cubane, which is in line with the observed difference in water oxidation activity. Moreover, buffer molecules such as phosphate ions were found to be able to coordinate to the cubane WOC, demonstrating possible inhibitory effects of such - in calculations usually omitted - ions. ${ }^{[13]}$

After elucidating the catalytic resting state, we explored possible water oxidation mechanisms ${ }^{[7-9]}$ with emphasis on the commonly accepted nucleophilic water attack and oxo-oxo radical coupling mechanisms (and modifications thereof). (Approximate) barriers between certain states during the catalytic cycle were computed using minimum energy path calculations where inclusion of explicit solvent molecules again turned out to be crucial for capturing certain effects such as the ability of the solvation shell to mediate a proton transfer from the nucleophile to a basic site at the catalyst. Moreover, spin states were found to have a significant influence on the energetics of the catalytic cycle. The cubane core plays an important role by, for instance, flexible distribution of electrons over the core. Comparing the $\left\{\mathrm{Co}_{4}(\mathrm{OR})_{4}\right\}$ WOC with the $\left\{\mathrm{Co}_{3} \mathrm{Ln}(\mathrm{OR})_{4}\right\}(\mathrm{Ln}=\mathrm{Er}, \mathrm{Tm})$ cubanes, we have found that the latter are significantly more flexible. This concerns not only the ligand shell but also charge and protonation states, distribution of electrons and spin density, and even the cubane cage itself. The latter can change giving rise to so-called 'distorted' [9] and 'open' cubane

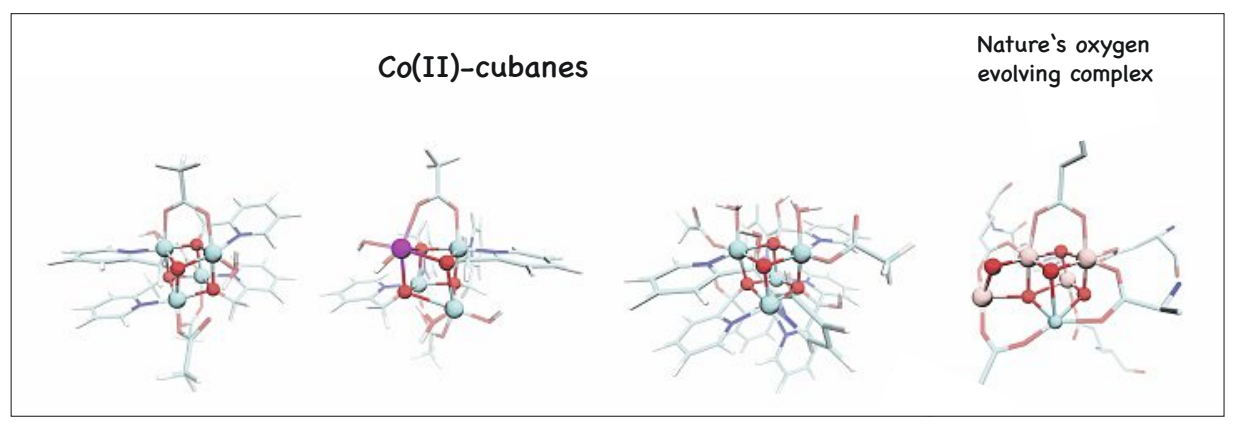

Fig. 3. From left to right: The first generation, $\left\{\mathrm{Co}_{4}(\mathrm{OR})_{4}\right\}(\mathrm{R}=\mathrm{hmp}),{ }^{[30]}$ second generation, $\left\{\mathrm{Co}_{3} \mathrm{Ln}(\mathrm{OR})_{4}\right\}(\mathrm{Ln}=\mathrm{Er}, \mathrm{Tm}, \mathrm{Yb}, \mathrm{Ho}),{ }^{[13]}$ and third generation, $\left[\mathrm{Co}_{4}{ }_{4}\left((\mathrm{dpy}(\mathrm{OH})(\mathrm{O}))_{4}\right)(\mathrm{OAc})_{2}\left(\mathrm{H}_{2} \mathrm{O}\right)_{2}\right]^{2+}$ $\left[\mathrm{dpy}(\mathrm{OH})(\mathrm{O})=\right.$ di-2-pyridinylmethanediolat] ${ }^{\left[{ }^{[1]}\right]}$ of the $\mathrm{Co}(\mathrm{II})$-based cubanes as well as the oxygen-evolving complex of photosystem II. ${ }^{[32]}$

structures, ${ }^{[7]}$ accompanied by changes in oxidation state (redox isomerism) and coordination environment. This highlights the fact that the structural framework of such catalysts can be very flexible, which might be of high relevance for catalytic activity and long-term stability. Redox isomerism and open cubane structures have also been observed for nature's OEC.[33] Our findings are thus, to the best of our knowledge, the first example for open cubane core structures of artificial WOCs. It is worth mentioning that proper inclusion of solvation has played a crucial role in the discovery and stabilisation of these opened cubane cages. The calculated water oxidation mechanisms for the $\left\{\mathrm{Co}_{3} \mathrm{Ln}(\mathrm{OR})_{4}\right\}$ $(\mathrm{Ln}=\mathrm{Er}, \mathrm{Tm})$ cubanes were found to have a lower overpotential than the ones calculated for the $\left\{\mathrm{Co}_{4}(\mathrm{OR})_{4}\right\}$ WOC, in accordance with experimentally measured differences in catalytic activity. Moreover, an additional advantageous influence of the redox-inert metal centre was detected during the nucleophilic water attack where a hydroxyl ligand bound to the Ln centre acted as a base to take up a proton from the attacking water molecule, in this way facilitating the attack. ${ }^{[9]}$ This is reminiscent of a recently emerging design concept for WOCs to include an intramolecular base for higher efficiency. Our findings suggest that the interplay of these various factors lay the basis for the higher catalytic activity observed for the $\left\{\mathrm{Co}_{3} \mathrm{Ln}(\mathrm{OR})_{4}\right\}(\mathrm{Ln}=\mathrm{Er}$, $\mathrm{Tm})$ cubanes compared to the $\left\{\mathrm{Co}_{4}(\mathrm{OR})_{4}\right\}$ WOC.

The latest generation of $\mathrm{Co}(\mathrm{II})$-based cubanes has two aqua ligands at the same face of the cubane cage (see Fig. 3), in this way mimicking the surface of heterogeneous cobaltoxide catalysts. Moreover, it was demonstrated that $\mathrm{Co}$ (II) metal centres can be replaced by $\mathrm{Ni}(\mathrm{II})$ ions. ${ }^{[31]}$ AIMD calculations revealed that the core of this recently presented $\mathrm{Co}$ (II)-based WOC is structurally flexible but no decomposition of the WOC had been observed. Beyond that, the protonation states of that WOC in solution have been found to change easi- ly due to the complex interaction with the surrounding water solvent and the hydrogen bonding network. ${ }^{[31]} \mathrm{A}$ recent review about computational challenges in catalysis with focus on Co-based water oxidation can be found in ref. [34].

\section{Informed Design of Water Oxidation Catalysts}

The study of bio-inspired Co(II)-based cubane WOCs has delivered essential knowledge for the derivation of structureactivity relationships. The information about the thermodynamics of the catalytic cycle and barriers connecting catalytic states as well as relevant side reactions can be used as a basis to improve the energetic performance of said processes. Aspects mentioned already in section 3 are spin states and flexibility with respect to ligand shell, cubane cage, protonation and oxidation states. ${ }^{[7,9]}$ In order to deduce principles for improving the thermodynamics, we have adapted the concept of a thermodynamically ideal catalyst which has the free energy associated with water oxidation equally distributed among the steps during the catalytic cycle, thus minimizing the overpotential of that process (see Fig. 4). ${ }^{[3,7-9,35]}$ This shows immediately what steps during the catalytic cycle may possibly be improved with respect to the thermodynamics. Lowering the barriers between the catalytic states, in particular in-between the rate-determining states, allows to tune the kinetics of the catalytic process. For instance, reducing the energy of an energetically low lying unoccupied molecular orbital with proper symmetry governing the nucleophilic water attack by suitable ligands can decrease the barrier associated with the attack. For the oxygen evolution step, tuning the $\Pi *$ orbitals in order to optimize the strength of the $\mathrm{Co}-\mathrm{OO}$ bond is another possibility. ${ }^{[8]}$

For derivation of other basic design guidelines, it is advantageous to study simpler systems with solely one or two tran- 


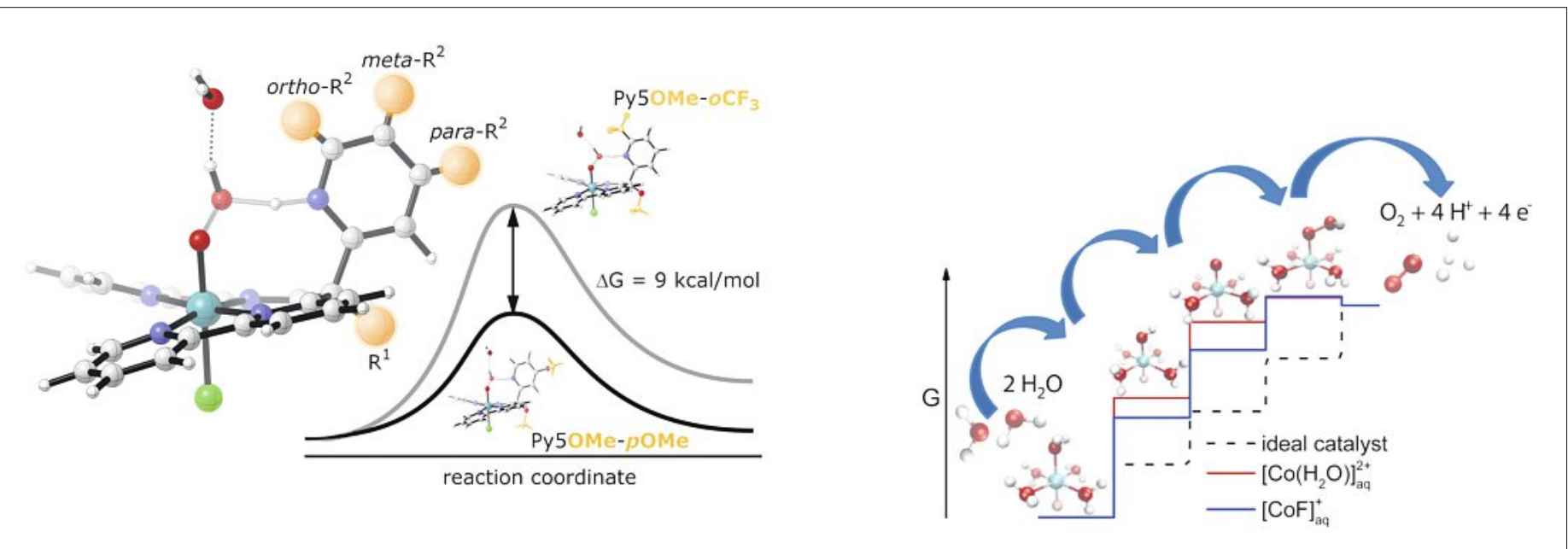

Fig. 4. Design of novel Ru-based WOCs (left; adapted from ref. [3]) and optimizing the thermodynamics of water oxidation for Co(II)-based model systems (right; adapted from ref. [35]).

sition metal centre(s). Inspired by experimental investigations of cobalt salts ${ }^{[36]}$ for water oxidation, we investigated a smaller model system, a cobalt aqua complex, in more detail (see Fig. 4). Systematically varying the ligand shell by introducing halide and pyridine (and derivatives thereof) ligands made clear how certain electrondonating or electron-withdrawing ligands can affect the thermodynamic catalytic performance and how the unoccupied molecular orbital fostering the nucleophilic water attack can be influenced.[35]

A heavily studied transition metal for water oxidation is ruthenium, and a large variety of mono- and dinuclear Ru-WOCs have been presented (for reviews, see for example refs. [37, 38]). For these catalysts, again an important question is the water oxidation mechanism, i.e. either a nucleophilic water attack or coupling of two oxo species. The ligands have a decisive influence on the mechanism. Subtle changes in the ligand framework can have a large impact on the energetics of the water oxidation mechanism and can lead to a switch between the two above-mentioned basic mechanisms as well. In close collaboration with experimental groups, we have, for instance, studied possible water oxidation pathways of two WOCs featuring the non-planar tetradentate ligand biisoquinoline dicarboxylic acid.[39] Other ligand frameworks were studied in refs. [2, 3], namely Ru-WOCs with a pentapyridine ligand composed of a pyridine and two bipyridine subunits linked together at an $\mathrm{sp}^{3}$ carbon atom, which has a fourth substituent (methyl or methoxy group) giving rise to different catalytic activities. Our calculations allowed to rationalize the differences in measured catalytic activities. Especially the step preparing the active species for the water oxidation catalysis (exchange of pyridine by water) has been found to be a crucial step determining the catalytic performance, ${ }^{[2]}$ not, as one would expect, the nucleophilic water attack forming the oxygen-oxygen bond. Moreover, we could rationalize why chloride as the sixth coordination ligand is essential for a high catalytic activity by identifying possible decomposition pathways. The various factors influencing the water oxidation activity were explored by modifications at the $\mathrm{sp}^{3}$ carbon atom and the pyridine ligand (see Fig. 4) using, e.g. dissociation scans. Moreover, we showed how quantities such as spin density and the energy of the lowest unoccupied molecular orbital correlate with different activation energies observed for varying substituents in the ortho, meta, and para position of the pyridine, respectively. ${ }^{[3]}$ This provides a promising way for computationally cheap access to such information without explicit calculation of transition states, which is in particular attractive if a large number of calculations have to be carried out such as in screening approaches or elucidation of reaction networks.

\section{Conclusion}

We have presented an overview about our recent work on computational methods and their application in catalysis. Current research for the former is manifold: On the one hand, we have worked on the application of forefront multiconfigurational approaches for a highly accurate electronic structure beyond DFT, which paves the way to assess possible shortcomings of DFT and to gain deeper understanding on the role of complex transition metals and their ligands in catalytic processes. On the other hand, we have shown that environmental and dynamic effects often play an essential role, which are usually hardly covered in conventional static computational approaches. We therefore have also focused on AIMD-based methods for a sophisticated description of the environment, inclusion of solvent effects, as well as protocols to reliably calculate relevant properties such as redox potentials and free energies. Another pivotal field of research has been spectroscopy where we have presented a wide variety of developments ranging from periodic subsystem DFT over elucidation of excited states to (chirality- and interface-sensitive) vibrational and X-ray spectroscopic methods.

Emphasis regarding the applications described has been laid on water oxidation catalysis as one of the main limiting part for artificial water splitting devices. In particular, we have investigated $\mathrm{Co}$ (II)-based cubane WOCs, which are some of the rare stable and active cubane WOCs mimicking nature's cubane WOC and thus ideal model systems to elucidate factors influencing bio-inspired water oxidation in detail. Investigation of environmental effects such as the impact of solvent and buffer ions in solution has made clear that interactions of the latter with the catalyst can significantly alter their behaviour and should be accounted for in the calculations in a sophisticated way. Study of water oxidation mechanisms has provided additional insight revealing several facets promoting efficient water oxidation. In particular, great flexibility of the Co(II)-based cubanes has been observed. This is especially true for the $\mathrm{Co}(\mathrm{II})$-cubanes containing a redox-inert lanthanide metal centre, which have been found to be flexible with respect to the ligand shell, the cubane cage (associated with change in coordination environment and oxidation state), and protonation states in addition to intramolecular base functionality. Moreover, the calculations suggest several routes how to improve the catalytic behaviour of such bio- inspired Co(II)-cubanes. Ongoing work also focuses on the elucidation of water oxidation 
mechanisms of the latest generation of Co(II)-cubanes as surface mimics of heterogeneous cobalt-oxide catalysts.

Besides the involved cubane WOCs, we have investigated several mononuclear model systems and Ru-based WOCs. Systematically modifying the ligand shell allowed us to discover trends how to ameliorate the thermodynamics and/or kinetics of the water oxidation process and what easily accessible computational quantities correlate with that. In addition, exploring side reactions such as decomposition pathways has been important for an overall picture. The in-depth information gained by means of forefront computational approaches demonstrates that calculations are indispensable for an in-depth study of water oxidation processes and will become even more important in the future, paving the way for derivation of highly soughtfor structure-activity relationships and informed in silico design, in the field of catalysis and beyond.

\section{Acknowledgment}

The author thanks all previous and current group members who contributed to the work mentioned in this article and whose names can be found in the corresponding references. Moreover, the author is grateful to Mauro Schilling for support in preparing the figures and reading the manuscript as well as the experimental groups of Greta R. Patzke (UZH), Roger Alberto (UZH), and Antoni Llobet (ICQC) for the collaborations. Funding by the University of Zurich, the Swiss National Science Foundation (grant no: PP00P2 170667), and the University Research Priority Program LightChEC is gratefully acknowledged. We thank the Swiss National Supercomputing Center for computing resources (project ID: s502, s745, and s788).
[1] G. Li Manni, S. D. Smart, A. Alavi, J. Chem. Theory Comput. 2016, 12, 1245.

[2] M. Gil-Sepulcre, M. Böhler, M. Schilling, F. Bozoglian, C. Bachmann, D. Scherrer, T. Fox, B. Spingler, C. Gimbert-Surinach, R. Alberto, X. Sala, S. Luber, C. J. Richmond, A. Llobet, ChemSusChem 2017, 10, 4517.

[3] M. Schilling, M. Böhler, S. Luber, Dalton Trans. 2018, DOI:10.1039/c8dt01209a.

[4] M. Böhler, Master thesis, University of Zurich, 2017.

[5] F. Hodel, P. Deglmann, S. Luber, J. Chem. Theory Comput. 2017, 13, 3348.

[6] D. Marx, J. Hutter, 'Ab Iinito Molecular Dynamics: Basic Theory and Advanced Methods' Cambridge University Press: Cambridge, 2009.

[7] M. Schilling, F. H. Hodel, S. Luber, ChemSusChem 2017, 10, 4561.

[8] F. Hodel, S. Luber, ACS Catal. 2016, 6, 1505.

[9] F. Hodel, S. Luber, ACS Catal. 2016, 6, 6750.

[10] A. Klamt, G. Schüürmann, J. Chem. Soc. Perkin Trans. 2 1993, 5, 799.

[11] F. Eckert, A. Klamt, AIChE J. 2002, 48, 369.

[12] A. Klamt, Wiley Interdiscipl. Rev. Comput. Mol. Sci. 2011, 1, 699 .

[13] F. Evangelisti, R. Moré, F. Hodel, S. Luber, G. R. Patzke, J. Am. Chem. Soc. 2015, 137, 11076.

[14] A. Laio, M. Parrinello, Proc. Natl. Acad. Sci. U.S.A. 2002, 99, 12562.

[15] F. Hodel, S. Luber, J. Chem. Theory Comput. 2017, 13, 974.

[16] J. Cheng, X. Liu, J. VandeVondele, M. Sulpizi, M. Sprik, Acc. Chem. Res. 2014, 47, 3522.

[17] S. Luber, Chimia 2018, 72, 328.

[18] S. Luber, Habilitation thesis, University of Zurich, 2016.

[19] S. Luber, J. Chem. Phys. 2014, 141, 234110

[20] S. Luber, J. Phys. Chem. A 2013, 117, 2760.

[21] S. Luber, M. Iannuzzi, J. Hutter, J. Chem. Phys. 2014, 141,094503 .

[22] S. Luber, Biomed. Spectrosc. Imaging 2015, 4, 255.

[23] S. Luber, J. Chem. Theory Comput. 2017, 13, 1254.

[24] S. Luber, J. Phys. Chem. Lett. 2016, 7, 5183.

[25] Y. Gurdal, S. Luber, J. Hutter, M. Iannuzzi, Phys. Chem. Chem. Phys. 2015, 17, 22846.

[26] P. Oulevey, S. Luber, B. Varnholt, T. Bürgi, Angew. Chem. Int. Ed. 2016, 55, 11787.
[27] S. Luber, K. Adamczyk, E. T. J. Nibbering, V. S Batista, J. Phys. Chem. A 2013, 117, 5269.

[28] S. Luber, I. Rivalta, Y. Umena, K. Kawakami, J.-R. Shen, N. Kamiya, G. W. Brudvig, V. S. Batista, Biochemistry 2011, 50, 6308.

[29] S. Luber, S. Leung, C. Herrmann, W. Han Du, L. Noodleman, V. S. Batista, Dalton Trans. 2014, 43, 576.

[30] F. Evangelisti, R. Güttinger, R. Moré, S. Luber, G. R. Patzke, J. Am. Chem. Soc. 2013, 135, 18734.

[31] F. Song, R. Moré, M. Schilling, G. Smolentsev, N. Azzaroli, S. Luber, G. R. Patzke, J. Am. Chem. Soc. 2017, 139, 14198.

[32] Y. Umena, K. Kawakami, J.-R. Shen, N Kamiya, Nature 2011, 473, 55.

[33] D. A. Pantazis, W. Ames, N. Cox, W. Lubitz, F. Neese, Angew. Chem. Int. Ed. 2012, 51, 9935.

[34] M. Schilling, S. Luber, Front. Chem. 2018, 6, 100.

[35] M. Schilling, G. R. Patzke, J. Hutter, S. Luber, J. Phys. Chem. C 2016, $120,7966$.

[36] H. Liu, M. Schilling, M. Yulikov, S. Luber, G. R. Patzke, ACS Catal. 2015, 5, 4994.

[37] M. D. Kärkäs, B. Åkermark, Dalton Trans. 2016, 45,14421

[38] J. D. Blakemore, R. H. Crabtree, G. W. Brudvig, Chem. Rev. 2015, 115, 12974.

[39] D. Scherrer, M. Schilling, S. Luber, T. Fox, R Alberto, C. J. Richmond, Dalton Trans. 2016, 45, 19361. 